\title{
Dielectric and Mechanical Properties Evaluation of Polypropylene Containing Nitride-based Nanoparticles
}

\author{
Zheng Lian, Xinyu Wang and Thomas Andritsch \\ The Tony Davies High Voltage Laboratory \\ University of Southampton \\ Southampton, UK
}

\begin{abstract}
Polypropylene (PP) which is recyclable and easy to process, has the potential to be a promising alternative for conventional cross-linked polyethylene (XLPE) for using in high voltage power cable insulation. This paper aims to determine the effects of different types of nitride-based nanoparticles on the dielectric and mechanical properties of PP. Thus, three nitridebased fillers, boron nitride, aluminium nitride and silicon nitride are selected. To evaluate the influence of different filler concentrations, PP nanocomposite systems containing 5 or 10 wt.\% of nitride-based nanoparticles were prepared by a modified solution blending method. Meanwhile, $5 \mathrm{~g}$ of neat PP was made with the same procedure to be the reference system. For determining the most suitable fillers in terms of electromechanical properties, three experiments namely, AC breakdown, dielectric spectroscopy and tensile test were conducted. The results of these experiments indicate PP with 5 wt.\% of boron nitride has the best performance on dielectric and mechanical properties among nanocomposites.
\end{abstract}

Keywords- Polypropylene, Nitride-based nanoparticles, AC breakdown, Dielectric spectroscopy, Tensile strength

\section{INTRODUCTION}

All electric apparatuses rely on insulation and as the level of power system capacity increasing, the demand placed on highperformance and cost-effective insulation is becoming more relevant [1]. For conventional high voltage cable insulation, XLPE has been widely used today. However, XLPE has some intrinsic drawbacks, such as the limited operation temperature $\left(90^{\circ} \mathrm{C}\right)$ and poor recyclability, which restrict it for further applications [2]. PP has been an active area of research due to its recyclability and promising dielectric characteristics. The melting point of PP is around $170{ }^{\circ} \mathrm{C}$ which is much higher than the maximum working temperature of XLPE-based cables. Additionally, PP copolymer has the potential to inhibit space charge [2]. However, there do exist some drawbacks of PP, including low thermal conductivity and high brittleness $[2$, 3].

Many researchers reported that dielectric and thermal properties of PP can be enhanced by adding nano-fillers [3, 4]. However, oxide-based nano-fillers suffer from water absorption which decreases the dielectric performance of nanocomposites $[5,6]$. By contrast, nitride-based nano-fillers with less hydrophilic sites can minimize the effects of water absorption [1,5]. Additionally, nitride-based nanoparticles possess promising properties, such as high electrical resistivity, high thermal conductivity and low thermal expansion coefficient which could potentially improve the performance of PP [3]. In this paper, efforts are made to improve the mechanical properties, while, maintaining or improving the dielectric properties of PP.

\section{EXPERIMENTAL}

\section{A. Materials and Sample Preparation}

Isotactic polypropylene was purchased from Sigma-Aldrich (427861). Three types of nitride-based nanoparticles are used. Boron nitride (BN, NX1 $900 \mathrm{~nm}$ ), aluminium nitride (AlN, 50 $\mathrm{nm})$ and silicon nitride $(\mathrm{SiN},<50 \mathrm{~nm})$ were obtained from Momentive Performance Materials, Aladdin Inc. and SigmaAldrich respectively.

A modified solvent blending method based on $[5,6,7]$. was applied. To obtain 5 or $10 \mathrm{wt} . \%$ nanocomposites, 4.75 or $4.5 \mathrm{~g}$ PP were weighted and dissolved in $50 \mathrm{ml}$ of xylene at $139{ }^{\circ} \mathrm{C}$. At the same time, 0.25 or $0.5 \mathrm{~g}$ of the nanoparticles were added in $10 \mathrm{ml}$ of toluene or isopropyl alcohol (IPA) and treated with a probe sonicator (Hielscher UP200S) for $30 \mathrm{~min}$. $\mathrm{BN}$ and $\mathrm{AlN}$ were added in toluene while SiN was added in IPA. The solvents were assumed not to impact the final measurements since xylene, toluene and IPA would be completely removed through the drying process used during sample preparation. When the sonication treatment on nanoparticles/toluene or IPA mixture was complete, the $\mathrm{PP} /$ xylene mixture was removed from the heat and the nanoparticles with toluene or IPA mixture was added immediately. The resulting mixture was stirred with a glass rod until it solidified. Subsequently, the resulting solid was cut into cubes using the glass stirring rod and dried in a fume cupboard for 2 days to remove most of the solvents. Afterwards, pieces of the resulting nanocomposites were pressed into sheets using a Micro-Mould $10 \mathrm{~T}$ and pressed at $180{ }^{\circ} \mathrm{C}$ by applying 3 tons pressure to remove potentially trapped air bubbles. The obtained sheet was cut into 4 pieces and then repeatedly pressed to obtain a uniformly distributed sample. In the end, the pressed samples were vacuum dried at $80{ }^{\circ} \mathrm{C}$ for 3 days to ensure the solvent was completely removed. The samples are denoted as "filler-loading ratio", e.g. $\mathrm{BN}-5$ is the PP nanocomposite that contains 5 wt. \% of $\mathrm{BN}$ filler.

\section{B. Characterisations}


AC breakdown tests were conducted on an AC breakdown kit according to ASTM standard D149-87. Disc-shaped samples of $4 \mathrm{~mm}$ in diameter and $85 \pm 10$ microns in thickness were made. 5 test points were marked on each sample and separated by at least $5 \mathrm{~mm}$ from each other and the edges of the sample to prevent surface flashover. The voltage increment rate was set to $100 \mathrm{~V} / \mathrm{s}$, the sample was placed between the electrodes and immersed in silicone oil. 20 valid data points of each system were recorded.

Dielectric spectroscopy was performed with a Solartron 1296 dielectric interface connected to a Schlumberger SI 1260 Impedance/phase gain analyser (ASTM standard D150-98). The sample holders of Solartron 1296 are two parallel discshaped electrodes with $32 \mathrm{~mm}$ in diameter. Disc-shaped samples of $40 \mathrm{~mm}$ in diameter and $230 \pm 10$ microns in thickness were prepared. The applied voltage was $3 \mathrm{~V}$ (AC) and there were two impedance measurement cycles. For the first cycle, the frequency swept from $100 \mathrm{kHz}$ to $1 \mathrm{~Hz}$ at 5 points per decade and 5 seconds integration per point. For the other cycle, the frequency swept from $1 \mathrm{~Hz}$ to $100 \mathrm{MHz}$ at 5 points per decade and 10 cycles integration per point.

The ultimate tensile strength was measured by a Tinius Olsen H25KS tester. Rectangular samples of $260 \pm 10$ microns in thickness were made. Dumbbell-shaped samples were not used due to limitations of the equipment. Each sample is of $5 \mathrm{~cm}$ in length and $1 \mathrm{~cm}$ in width. The rectangle samples were stretched at a constant extension rate of $9 \mathrm{~mm} / \mathrm{min}$ until they broke. Only reading from which samples tore near the centre and not at the clamping point were taken. The gauge length was kept at $3 \mathrm{~cm}$.

\section{RESULTS AND DISCUSSION}

\section{A. AC Breakdown Strength}

AC breakdown tests were conducted under room temperature and a 2-parameter Weibull distribution was used to analyse results. Fig.1 shows AC breakdown results of all nanocomposites. In terms of scale parameter, the result of unfilled PP is $197.48 \mathrm{kV} / \mathrm{mm}$. By adding different nanoparticles with different filler concentrations, Weibull plots show a change of breakdown strength between neat PP and different nanocomposites. For boron nitride nanocomposites, the scale parameters for $5 \mathrm{wt} . \%$ and $10 \mathrm{wt} . \%$ samples increase to $210.38 \mathrm{kV} / \mathrm{mm}$ and $204 \mathrm{kV} / \mathrm{mm}$, respectively. By contrast, $\mathrm{AlN}$ and $\mathrm{SiN}$ show a detrimental effect on AC breakdown strength. For aluminium nitride filled samples, the AC breakdown strength decreases from 161.97 $\mathrm{kV} / \mathrm{mm}$ to $143.32 \mathrm{kV} / \mathrm{mm}$ by increasing the filler concentration from $5 \mathrm{wt} \%$ to $10 \mathrm{wt}$. \%, while the $\mathrm{SiN}$ filled samples decrease from $123.09 \mathrm{kV} / \mathrm{mm}$ to $72.08 \mathrm{kV} / \mathrm{mm}$. Considering the shape parameter, the results of the unfilled PP is 16.39. However, the shape parameters of all nitride-based nanocomposites decline to varying degrees. All results of nanocomposites are around 10 which is much lower than neat PP. this may be related to the inhomogeneous dispersion of nano-fillers and the change of the morphology of PP.

Ayoob suggested the better electric breakdown performance after adding $\mathrm{BN}$ could be related to the layered platelets structure of $\mathrm{BN}$, which can act as physical barriers inhibiting the propagation of a breakdown path [1]. Another reason could be the morphology change, especially the interfacial regions, where the polymer is changed by additives. This can leads to an increased density of charge traps and changes the electrical breakdown strength $[1,4]$. Decreased breakdown strength after adding $\mathrm{AlN}$ and $\mathrm{SiN}$ could be related to hydrophilic sites, such as $-\mathrm{OH}$, on the surface of nanoparticles. The surface of AIN and SiN has much more hydrophilic sites compared to $\mathrm{BN}$. This hydrophilic sites can absorb water
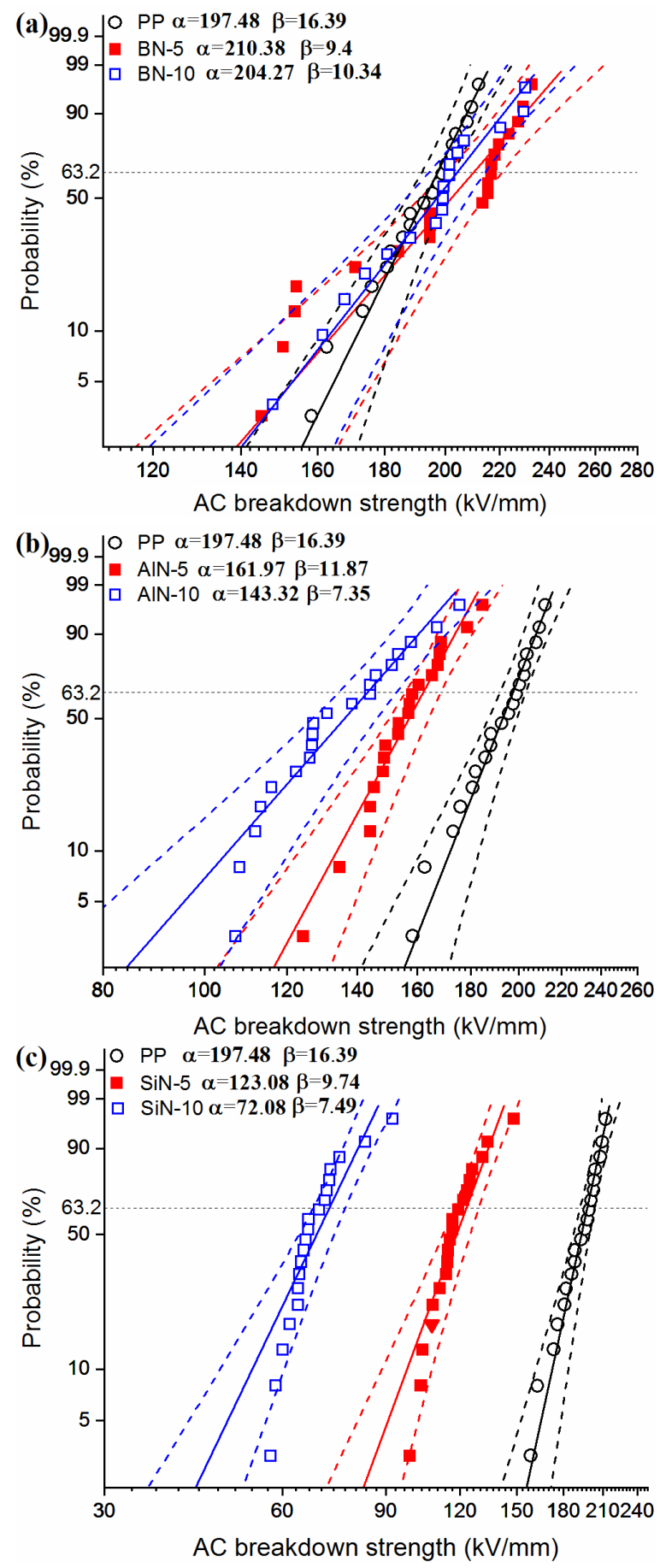

Fig. 1. AC breakdown strength of (a) BN/PP, (b) AlN/PP and (c) SiN/PP 
molecules from the surrounding environment and form conductive pathways which lead to uneven field distribution and conduction, resulting in lower breakdown strength $[1,6]$. Narrowing the scope to a single filler, the effects of filler concentrations are very consistent. The results of $5 \mathrm{wt} \%$ nanocomposites are always higher than its 10 wt.\% counterparts.

\section{B. Dielectric Spectroscopy}

The real and imaginary part of the permittivity shown in
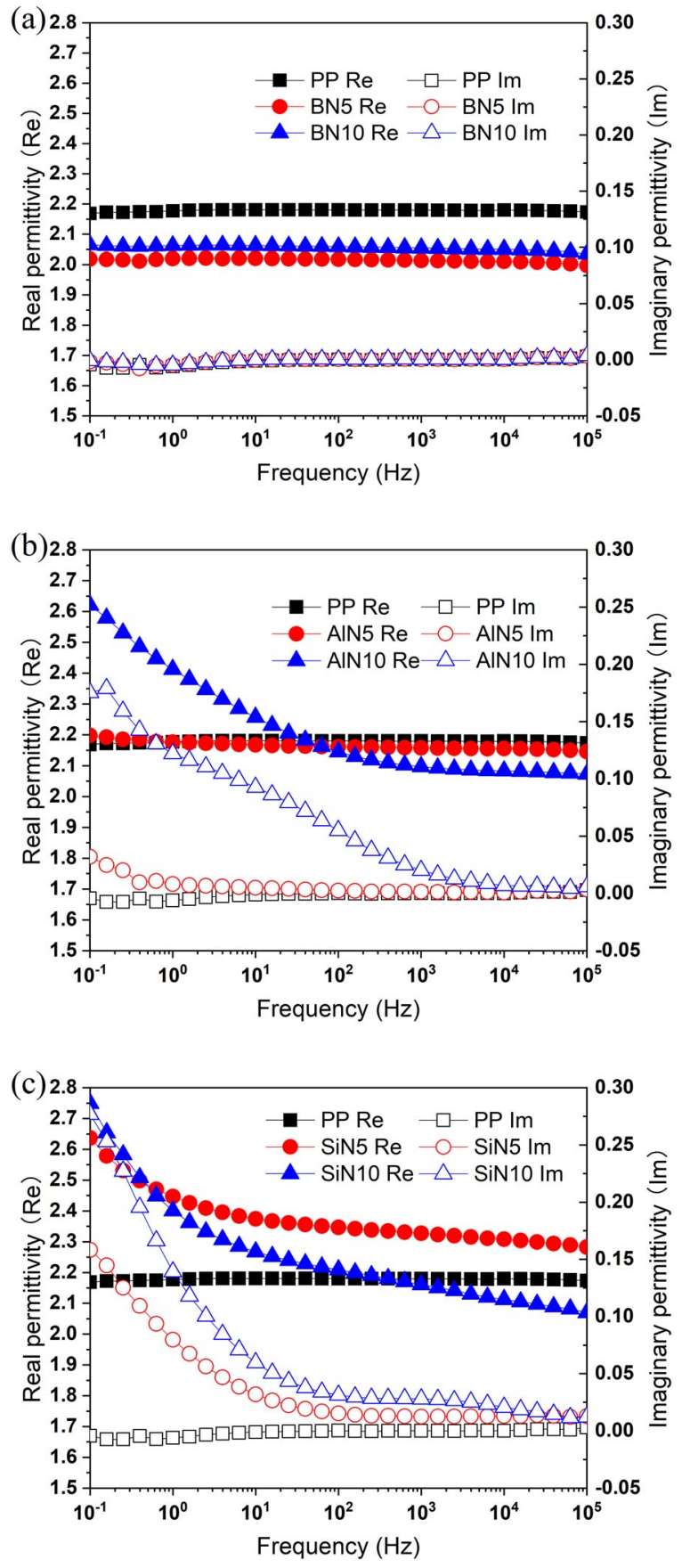

Fig. 2. Real and imaginary permittivity of (a) BN/PP, (b) AlN/PP and (c) $\mathrm{SiN} / \mathrm{PP}$
Fig. 2 and significant differences in different systems can be observed. For unfilled PP, the real part of the relative permittivity is frequency independent (around 2.17) with a negligible imaginary part of permittivity $\left(<10^{-3}\right)$. The lower real permittivity of boron nitride nanocomposites could be associated with the increased crystallinity of PP by adding BN which acts as nucleating agents that will lead to difficult reorientations of chain elements [4]. For AlN and SiN nanocomposites, lower real permittivity was observed in Higher filler loading nanocomposites, at $\sim 100$ to $10^{5} \mathrm{~Hz}$ and 0.5 to $10^{5} \mathrm{~Hz}$, respectively. The reason behind this phenomenon could be related to the higher filler loading leading to increased interactions between the polypropylene matrix and nanoparticles, which inhibits the mobility of the polymer chains [8]. A clear increase of the real part of the relative permittivity from high frequency to low frequency was observed in AlN and SiN systems, which could be related to water absorption and the interfacial polarization $[1,5,6]$.

For BN nanocomposites, as BN with a hydrophobic surface which can only absorb a very limited amount of water, no relaxation and upturn features at low frequency can be observed [1]. Relaxation processes can be observed in AlN-10 and $\mathrm{SiN}-10$ at about $0.1 \mathrm{kHz}$ and $1 \mathrm{kHz}$, respectively. This can be related to the absorbed water inside the samples $[1,5,6]$. The observed low-frequency dispersion in AlN and $\mathrm{SiN}$ samples could also be related to Maxwell-Wagner interfacial polarization or to the increased conductivity [1]. It worth to mention that the result of AlN-10 is very different from previous work where no relaxation process can be observed at the tested frequency range even at $90{ }^{\circ} \mathrm{C}$ [7]. The irreproducible results here can be related to the hydrolytic instability of AlN in moist air. The results of Li et al. indicates AIN is really sensitive to the water in the ambient environment and the hydrolytic instability also very depends on the manufacturing method. AlN manufactured through chemical vapour deposition from triethyl aluminium can gain over $80 \%$ of weight through irreversible hydrolytic degradation in moist air (80 RH \%) after 200 hours [9]. The hydrophilic aluminium hydroxide is formed after hydrolytic degradation, which can absorb water and possibly form a water shell surround the particle. This process is unavoidable during the manufacturing, packaging, and storage of the AlN nanoparticles. This will lead to the as-received AlN to have a variable range of surface chemistry, which can result in the inconsistent results compared to previously measured the AlN filled samples.

\section{Tensile Strength}

All tensile strength tests were carried out on the same day and under controlled temperature via air conditioning with same settings to avoid effects of temperature and humidity. Stress-strain curves were used to analyse the mechanical properties of PP and nitride-based nanocomposites. The results of stress-strain curves of all nanocomposites and unfilled PP are shown in Fig.3.

To ensure the flexibility of cable transportation and installation, materials with lower elastic modulus are the 


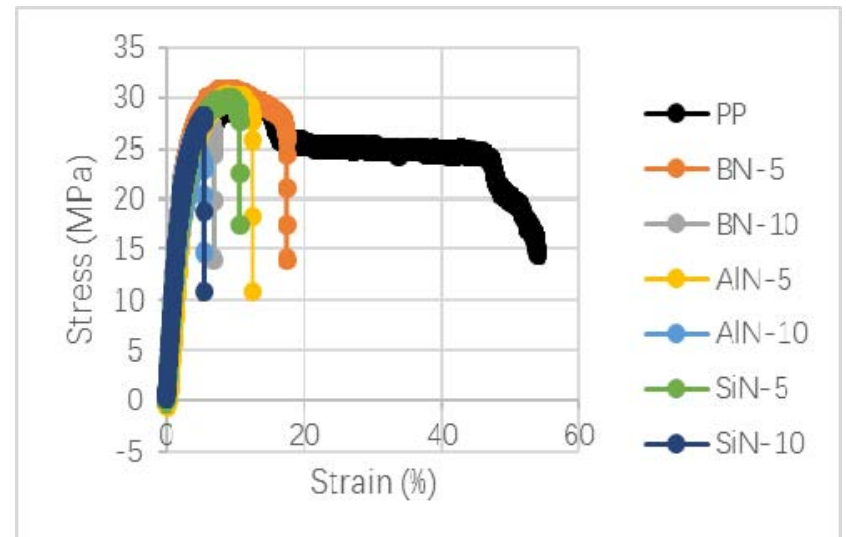

Fig. 3. Stress-Strain curves of all nanocomposites and unfilled PP

TABLE I

Young's Modulus of Nitride-BASEd Filler/PP Nanocomposites AND UNFILLED PP

\begin{tabular}{|c|c|}
\hline Sample & Young's Modulus (MPa) \\
\hline PP & 280.95 \\
\hline BN-5 & 391.86 \\
\hline BN-10 & 512.96 \\
\hline AlN-5 & 354.04 \\
\hline AlN-10 & 487.03 \\
\hline SiN-5 & 328.57 \\
\hline SiN-10 & 526.12 \\
\hline
\end{tabular}

preferred choice. Young's moduli of all tested samples are shown in Table I to judge the mechanical performance of each material. Throughout all samples, it is apparent that adding nitride-based nanoparticles to neat PP has negative impacts on the mechanical properties. For pure PP, the stress drops to drawing stress after reaching the yielding point and then remains almost constant until the breaking of the material [10]. However, all nanocomposites reach the instability limit after achieving the yielding point. Additionally, The stiffness of the material increases with the content of nanoparticles increasing, which could be related to the change in crystallinity [11].

Among all nano-fillers used in this project, 5 wt.\% of BN has the most beneficial impact on mechanical properties compared to other combinations.

\section{CONCLUSIONS}

PP possesses high breakdown strength, high melting point and recyclability, which is a great potential for high voltage cable insulating materials.

By adding different types of nitride-based fillers, the changes in the dielectric and mechanical properties of isotactic polypropylene are different. BN fillers enhance the dielectric performance of PP while AIN and SiN fillers lead to a worse dielectric result. This reveals that not all nitride-based fillers are suitable for improving the dielectric property of PP and the dielectric properties are highly related to the surface chemistry and shape of fillers. In addition, all nitride-based fillers investigated have a negative impact on the tensile strength of PP.

Among all investigated nitride-based fillers with variable filler concentrations, $\mathrm{BN}-5$ has the optimal performance as it increases the AC breakdown strength of pure PP (from $197.48 \mathrm{kV} / \mathrm{mm}$ to $210.38 \mathrm{kV} / \mathrm{mm}$ ) and even achieves a lower relative permittivity (around 2) compared with neat PP (around 2.2) while slightly increases the Young's modulus of unfilled PP (from 280.95 MPa to $391.86 \mathrm{MPa}$ ).

\section{ACKNOWLEDGMENT}

The authors would like to acknowledge the support of Professor George Chen and Mr. Orestis Vryonis. All data supporting this study are from Zheng Lian's dissertation for MSc Energy and Sustainability with Electrical Power Engineering submitted to University of Southampton.

\section{REFERENCES}

[1] R. Ayoob, "Dielectric properties of hexagonal boron nitride polymer nanocomposites," University of Southampton, 2017.

[2] X. Huang, Y. Fan, J. Zhang, and P. Jiang, "Polypropylene Based Thermoplastic Polymers for Potential Recyclable HVDC Cable Insulation Applications," IEEE Trans. Dielectr. Electr. Insul., vol. 24, no. 3, pp. 1446-1456, 2017.

[3] X. Huang, P. Jiang, and T. Tanaka, "A review of dielectric polymer composites with high thermal conductivity," IEEE Electr. Insul. Mag., vol. 27, no. 4, pp. 8-16, 2011.

[4] W. Cao, Z. Li, G. Sheng, and X. Jiang, "Insulating Property of Polypropylene Nanocomposites Filled with Nano-MgO of Different Concentration," IEEE Trans. Dielectr. Electr. Insul., vol. 24, no. 3, pp. 1430-1437, 2017.

[5] I. L. Hosier, M. Praeger, A. S. Vaughan, and S. G. Swingler, "The effects of water on the dielectric properties of aluminum based nanocomposites," IEEE Trans. Nanotechnol., vol. PP, no. 99, pp. 1-10, 2016.

[6] I. L. Hosier, M. Praeger, A. S. Vaughan, and S. G. Swingler, "The effects of water on the dielectric properties of silicon based nanocomposites," IEEE Trans. Nanotechnol., vol. PP, no. 99, pp. 1-10, 2016.

[7] X. Wang, T. Andritsch, and G. Chen, "Effect of surface functionalization on the dielectric properties of Polypropylene Aluminium Nitride Nanocomposites," in IEEE Int. Conf. on Dielectr. (ICD), 2018.

[8] Y. Hadajadj and R. A. Ghunem, "Dielectric Measurements on Polypropylene Nanocomposites Filled with Natural and Synthetic Nanoclay," in IEEE Conf. Electr. Insul. Dielectr. Phenom. (CEIDP), 2017, pp. 465-468.

[9] J. Li, M. Nakamura, T. Shirai, K. Matsumaru, C. Ishizaki, and K. Ishizaki, "Mechanism and kinetics of aluminum nitride powder degradation in moist air," J. Am. Ceram. Soc., vol. 89, no. 3, pp. 937943, 2006.

[10] A.-H. I. Mourad, "Thermo-mechanical characteristics of thermally aged polyethylene/polypropylene blends," Mater. Des., vol. 31, no. 2, pp. 918-929, 2010.

[11] K. Zhang, L. Li, L. Zhong, N. Chen, M. Xu, D. Xie, and G. Chen, "The Mechanical Properties of Recyclable Cable Insulation Materials Based on Thermo-plastic Polyolefin Blends," in IEEE Int. Conf. Prop. Appl. Dielectr. Mater. (ICPADM), 2015, pp. 532-535. 